\title{
The incubation period of COVID-19: a global meta-analysis of 53 studies and a Chinese observation study of 11545 patients
}

\author{
Cheng Cheng ${ }^{1}$, DongDong Zhang ${ }^{2}$, Dejian Dang 3 , Juan Geng ${ }^{1}$, Peiyu Zhu ${ }^{1}$, Mingzhu Yuan ${ }^{1}$, Ruonan Liang ${ }^{1}$, \\ Haiyan Yang ${ }^{1}$, Yuefei Jin ${ }^{1}$, Jing Xie ${ }^{4,5}$, Shuaiyin Chen ${ }^{1 *}$ and Guangcai Duan ${ }^{1,4^{*}}$
}

\begin{abstract}
Background: The incubation period is a crucial index of epidemiology in understanding the spread of the emerging Coronavirus disease 2019 (COVID-19). In this study, we aimed to describe the incubation period of COVID-19 globally and in the mainland of China.

Methods: The searched studies were published from December 1, 2019 to May 26, 2021 in CNKI, Wanfang, PubMed, and Embase databases. A random-effect model was used to pool the mean incubation period. Meta-regression was used to explore the sources of heterogeneity. Meanwhile, we collected 11545 patients in the mainland of China outside Hubei from January 19, 2020 to September 21, 2020. The incubation period fitted with the Log-normal model by the coarseDataTools package.

Results: A total of 3235 articles were searched, 53 of which were included in the meta-analysis. The pooled mean incubation period of COVID-19 was 6.0 days (95\% confidence interval [CI 5.6-6.5) globally, 6.5 days (95\% Cl 6.1-6.9) in the mainland of China, and 4.6 days ( $95 \% C / 4.1-5.1)$ outside the mainland of China ( $P=0.006)$. The incubation period varied with age $(P=0.005)$. Meanwhile, in 11545 patients, the mean incubation period was 7.1 days (95\% Cl 7.0-7.2), which was similar to the finding in our meta-analysis.

Conclusions: For COVID-19, the mean incubation period was 6.0 days globally but near 7.0 days in the mainland of China, which will help identify the time of infection and make disease control decisions. Furthermore, attention should also be paid to the region- or age-specific incubation period.
\end{abstract}

Keywords: COVID-19, Incubation period, Meta-analysis

\section{Background}

Coronavirus disease 2019 (COVID-19) is caused by severe acute respiratory syndrome coronavirus (SARSCoV-2). It has spread over 223 countries and has been declared a pandemic on March 11, 2020 by World Health Organization [1]. The number of COVID-19 patients is

\footnotetext{
*Correspondence: sychen@zzu.edu.cn; gcduan@zzu.edu.cn

${ }^{1}$ Department of Epidemiology and Health Statistics, College

of Public Health, Zhengzhou University, No. 100 Kexue Avenue, Zhengzhou 450001, Henan, People's Republic of China

Full list of author information is available at the end of the article
}

rapidly increasing globally. More than 113.5 million confirmed cases and 2.5 million deaths were reported globally by March 2, 2021 [1]. The COVID-19 pandemic has become a worldwide public health issue.

The incubation period, known as the interval between initial infection and onset of disease, is an important index to characterize the spread of infectious disease and formulate quarantine measures. For example, the mean incubation period is often used to calculate the reproduction number, and the maximum incubation period is used to determine the duration of quarantine [2]. For COVID-19, original author(s) and the source, provide a link to the Creative Commons licence, and indicate if changes were made. The images or other third party material in this article are included in the article's Creative Commons licence, unless indicated otherwise in a credit line to the material. If material is not included in the article's Creative Commons licence and your intended use is not permitted by statutory regulation or exceeds the permitted use, you will need to obtain permission directly from the copyright holder. To view a copy of this licence, visit http://creativecommons.org/licenses/by/4.0/. The Creative Commons Public Domain Dedication waiver (http://creativeco mmons.org/publicdomain/zero/1.0/) applies to the data made available in this article, unless otherwise stated in a credit line to the data. 
its average incubation period has a wide range, ranging from 2.87 days [3] to 17.6 days [4]. Determining the duration of the quarantine is difficult. Several studies have provided mean incubation periods of COVID-19 of about 8 days in the mainland of China outside Hubei Province [5-7]. Some studies have reported shorter mean incubation periods of COVID-19 of about 5 days [8-10]. The incubation period of COVID-19 in previously published studies is inconsistent. In addition, few studies have focused on differences in the incubation period of COVID-19 worldwide. Only one study with 181 COVID-19 patients showed that the patients in the mainland of China had a shorter incubation period than others (4.8 days vs 5.5 days) [11], which seemed to contradict existing evidence. However, although previous meta-analyses have focused on the incubation period of COVID-19, the evidence on the region-specific incubation period is lacking. Therefore, an updated metaanalysis is necessary.

Although existing studies have presented the distribution of the incubation period of COVID-19 in China, they differ from one another probably because of the study population and estimation methods [12]. The first evidence on the incubation period of COVID-19 provides that the mean incubation period of COVID-19 is 5.2 days (95\% confidence interval $[C I] 4.1-7.0)$ in the early stage of the Wuhan epidemic [13]. The incubation period, defined as the time from the earliest exposure to onset, has a long distribution. Gao et al. revealed a mean incubation period of 9 days using this method [14]. Conversely, short incubation period was reported when it was defined as the time from the latest exposure to onset [8]. The above-mentioned methods inevitably overestimate or underestimate the incubation period of COVID-19. In general, the date of symptom onset is self-reported by the patient, which is considered exact data. Therefore, selecting COVID-19 patients with a single exposure would obtain an accurate incubation period set, which helps understand its distribution.

This study aimed to describe the incubation period of COVID-19. We conducted a meta-analysis to estimate the mean incubation period of COVID-19 globally and collected the information on COVID-19 patients in the mainland of China outside Hubei Province to understand its distribution. We selected patients with the precise date of infection and onset to calculate the accurate incubation period.

\section{Patients and methods}

\section{Systematic review and meta-analysis Literature search}

The meta-analysis was conducted on the basis of the Meta-analysis of Observational Studies in Epidemiology (MOOSE) group [15].
Two authors conducted a literature review on the incubation period of COVID-19 in CNKI, Wanfang, PubMed, and Embase databases between December 1, 2019 and May 26, 2021. The search terms combined incubation period (e.g., "incubation") and COVID-19 (e.g., "COVID19," “SARS-CoV-2," “2019-nCoV," "NCP," "Coronavirus disease 2019," "severe acute respiratory syndrome coronavirus 2," "novel coronavirus 2019," or "novel coronavirus pneumonia," Additional file 1: Table S1). The language was unrestricted. A total of 3235 articles were retrieved using the MeSH term and keywords.

\section{Selection criteria}

The irrelevant articles, reviews, and meta-analyses were excluded by title and abstract. Next, the remaining articles were read in detail. Eligible studies should include (1) the mean or median incubation period of COVID19; and (2) standard deviation $(S D)$, interquartile range (IQR), or range. Studies with a sample size of less than ten were excluded. If several eligible studies were identified from the same population, then we will select the article with the largest sample size. When $S D$ was not provided, the IQR and range were used to estimate the $S D[16,17]$. Finally, 53 eligible studies were included in the meta-analysis. Figure 1 presents the flowchart.

\section{Data extraction}

Information on the first author, country, age, gender, sample of the study, and definition of incubation period was collected. All regions were divided into two groups (the mainland of China and outside the mainland of China). The mean or median age of studies was divided into two groups ( $<40$ years vs $\geq 40$ years). Several estimation methods of incubation period in these eligible

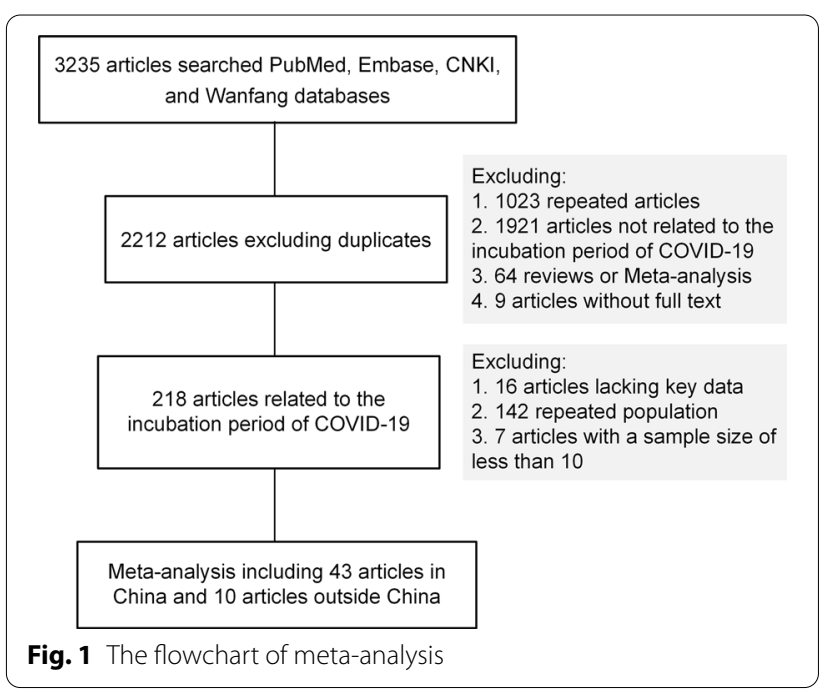


studies were identified, which were divided into three groups: (1) method 1 used the interval from the earliest date of exposure to the date of onset of symptoms to estimate incubation period; (2) method 2 used the interval between the exposure interval and the date of onset of symptoms to estimate the incubation period; (3) other methods.

Quality assessment tools to evaluate studies reporting the incubation period of infectious diseases were not available. Thus, the quality assessment of studies was waived.

\section{Estimations from confirmed COVID-19 patients in the mainland of China outside Hubei Province Study design and participants}

This study was approved by the Zhengzhou University Medical Ethics Committee (Zhengzhou, China). The information on all cases was collected from publicly available sources, and informed consent was waived.

By September 21, 2020, a total of 17168 patients with COVID-19 have been diagnosed with positive nucleic acid and clinical symptoms in the mainland of China outside Hubei Province. Among them, the available information of 11545 patients can be collected from the national and local health commissions in China [18]. We extracted patients' data, including age, sex, exposure history, dates of the nucleic acid positive, dates of onset systems, dates of the first treatment, dates of diagnosis, and official reports, to describe the characteristics of cases. All 11545 patients were included to estimate the incubation period.

We also screened 218 patients with the precise date of infection and onset from 11545 patients according to the following criteria to accurately calculate the incubation period in subgroup analysis: (1) a clear exposure history, such as contacting with a confirmed patient, traveling to Wuhan, or visiting a place with confirmed cases; (2) have a single short exposure (the earliest date of exposure $=$ the date of latest exposure); (3) have a clear date of exposure; and (4) have a clear date of onset systems (such as fever, cough, and fatigue). However, patients with only imaging results or positive nucleic acid were excluded. A total of 4242 patients had no clear date of onset; 11045 patients had no single short exposure or a clear date of exposure; and 74 patients had only symptoms of imaging result. Finally, a total of 218 patients met the screening criteria.

\section{Definition of case and variables}

A patient with positive nucleic acid and clinical symptoms was diagnosed as a COVID-19 case [19]. Highthroughput sequencing or real-time RT-PCR assay was used to detect nucleic acid in nasopharyngeal swab specimens.

The incubation period was defined as the time interval between the date of infection and the onset of disease. Among the 11045 patients, the onset of disease was clear in 7303 patients and unclear in 4242 patients. Previous evidence suggested that the mean time from onset to first treatment was 2.5 days, and the mean time from onset to diagnosis was 5.4 days [18]. Therefore, for the 4242 patients with an unclear date of onset, we defined date of onset as the date of first treatment minus 2.5 days $(n=2168)$ or the date of diagnosis minus 5.4 days $(n=2074)$. The date of infection was between the date of the earliest exposure and the date of the latest exposure. When the date of the earliest exposure was equal to the date of the latest exposure, the date of infection was the date of exposure; otherwise, it was represented as doubly interval-censored data [20]. Right exposure date (the latest exposure) was at least 1 day earlier than the date that nucleic acid positive or onset of disease was reported [12]. When it was not reported, right exposure date was defined as the date of the nucleic acid positive or the date of onset of disease minus 1 day. Considering most incubation periods of COVID-19 were shorter than 14 days, when the date of the earliest exposure was not reported, or the exposure interval was longer than 14 days, we assumed an exposure interval of 14 days.

\section{Statistical analysis}

In the meta-analysis, the mean incubation period and its $S D$ undergone a natural logarithmic transformation. Previous evidence suggested that the Log-normal distribution was the best fit for an incubation period of COVID-19 [18, 21, 22]. The log (incubation period) and its standard error were pooled using the DerSimonian and Laird random-effect model. Mean and $S D$ on the original scale were calculated from pooled estimates. Heterogeneity was quantified using the $I^{2}$ statistic and investigated by conducting subgroup analyses (such as the regions, average age of patients, ratio of male to female, and study sample). Meta-regression was used to compare differences in heterogeneity among subgroups. Begg's test and Egger's test were used to identify the publication bias.

We used mean $(95 \% C I)$ for continuous variables and count (percentage) for categorical variables. The distribution curve of the incubation period fitted with a Lognormal model by the "coarseDataTools" package [20]. Wilcoxon signed-rank test was used to compare differences in subgroup analysis by age and gender. A false discovery rate was used to adjust the $P$-value for multiple comparisons [23]. Sensitivity analysis explored the impact of different definitions of date of onset. 
Statistical analyses were conducted using $\mathrm{R}$ version 4.0.2 (R Foundation for Statistical Computing, Vienna, Austria) and STATA v12.0 (StataCorp LLC, Texas, USA). Two-sided $P<0.05$ indicated statistical significance.

\section{Results}

\section{Pooled mean incubation period in meta-analysis}

A total of 3235 articles were retrieved using the MeSH term and keywords. The authors identified 218 articles that reported the incubation period of COVID-19. Among them, 197 articles were from China, and four articles involved multiple countries. The other 17 articles were from Argentina [24], Brunei [25], Thailand [26], France [27, 28], Vietnam [29], Germany [30, 31], India [32, 33], Singapore [34, 35], Korea [3, 36, 37], Saudi Arabia [38], and Uganda [39]. There were 88 articles in English and 130 articles in Chinese (Fig. 1).

The meta-analysis included 53 articles, of which 43 were from China [40-83] and ten were outside China [3, 24-30, 32, 34] (Table 1). Figure 2 presents the forest plot of incubation periods. The pooled value was 6.0 days (95\% CI 5.6-6.5, $I^{2}=96.0 \%, P<0.001$ ) globally. In subgroup analysis, Table 2 and Additional file 2: Figures S1S8 showed the forest plot for the mean incubation period of COVID-19 by region, age, gender, sample size, estimation method of the date of infection, estimation method of the date of symptom onset, estimation method of the incubation period, and published language. The region, estimation method of the date of infection, and published language were significantly related to the incubation period of COVID-19. The incubation period was 6.5 days (95\% CI 6.1-6.9, $I^{2}=90.7 \%, P<0.001$ ) in the mainland of China and 4.6 days $\left(95 \%\right.$ CI $\left.4.1-5.1, I^{2}=83.3 \%, P<0.001\right)$ outside the mainland of China $\left(P_{\text {meta-regression }}<0.001\right)$. The short incubation period used the exposure interval (mean: 4.7 days; 95\% CI 3.1-7.0) from the earliest date of exposure (mean: 5.7 days; 95\% CI 5.0-6.4). A potential source of heterogeneity was not identified. Furthermore, no publication bias was identified in this meta-analysis (Additional file 3: Figure S9; both $P>0.05$ for Begg's test or Egger's test).

\section{Estimating the incubation period in the observation study}

Table 3 presents that the median age was 45 years (IQR: $33-56)$, and 5814 males (52.7\%) were included. The mean incubation period was 7.1 days $(95 \%$ CI $7.0-7.2)$ in the total population. Figure 3 shows that $5.4 \%$ of patients had an incubation period of less than 3 days; $10.2 \%$ of patients had an incubation period of more than 14 days, whereas $2.1 \%$ of patients had an incubation period of more than 21 days.

As shown in Table 3 and Additional file 4: Figure S10, the mean incubation period was 7.2 days $(95 \% C I$
7.0-7.4) in male and 7.1 days (95\% CI 6.9-7.3) in female $(P=0.603)$. Moreover, the mean incubation period was 8.6 days (95\% CI 8.0-9.3), 7.2 days (95\% CI 7.0-7.4), 7.1 days (95\% CI 6.9-7.2), and 7.0 days (95\% CI 6.6-7.3) in patients under 18 years, $18-40$ years, $41-60$ years, and over 60 years, respectively $(P<0.001)$.The incubation period was robust among different estimation methods of the date of onset $(P=0.319)$.

Table 3 presents the characteristics of 218 patients with a median age of 47 years (IQR: $35-56$ ); $56.3 \%$ of patients were men. The number of patients aged $<18$, $18-40,41-60$, and over 60 years was $6(2.8 \%), 73$ (34.1\%), 98 (45.8\%), and 37 (17.3\%), respectively. A total of 96 patients $(44.0 \%)$ were infected from January 19 to January 22 . Furthermore, a total of $65.6 \%$ of patients were exposed to confirmed cases.

Figure 4 shows the distribution of the accurate incubation period. The median incubation period was 7 days (range: 1-26 days; IQR: 5-11) in 218 patients. Twentyseven patients had an incubation period of more than 14 days. In the fitted model, the mean incubation period was 6.8 days (95\% CI 6.2-7.4), and $15 \%$ and $5.2 \%$ of patients had an incubation period of more than 14 and 21 days, respectively (Fig. 3).

In subgroup analysis (Additional file 5: Figure S11), the incubation period was shorter in patients aged 41-60 years than that in patients aged 18-40 years or over 61 years $(41-60$ years vs $18-40$ years: 6.0 days vs 9.0 days, adjusted $P=0.028 ; 41-60$ years vs over 60 years: 6.0 days vs 9.0 days, adjusted $P=0.041$ ). The patients exposed to confirmed cases had a longer incubation period than the other groups (9.0 days vs 6.0 days; $P=0.015)$. However, no significant difference in incubation period was observed with regard to sex or date of infection. The fitted curves showed a similar trend to the above-mentioned results.

\section{Discussion}

Several significant findings are obtained in our studies. First, this meta-analysis presented that the mean incubation period of COVID-19 was 6.0 days globally, but it was longer in the mainland of China than in other regions. Next, this study used a large sample to estimate the incubation period of COVID-19. For 11545 patients, the mean incubation period was 7.1 days; $10.2 \%$ and $2.1 \%$ of patients had an incubation period of more than 14 and 21 days. Similar results were found in 218 patients set with exact infection and onset data. Finally, only population studies showed that age was related to the incubation period of COVID-19.

The incubation period is a crucial parameter for understanding the epidemiological characteristics of COVID-19. In our study, the mean incubation period 
Table 1 Information on the studies included meta-analysis

\begin{tabular}{|c|c|c|c|c|c|c|}
\hline Author & Country & Case number & Age, years & Gender & Incubation period, days & References \\
\hline Ai et al & China & 44 & - & - & 8.09 (SD: 4.99) & [40] \\
\hline An et al & China & 27 & 44 & $11 \mathrm{M}: 16 \mathrm{~F}$ & 9.1 (R: 3-28) & [41] \\
\hline Chen et al & China & 19 & - & - & 5 (IQR: 3.3-10) & [42] \\
\hline Chen et al & China & 18 & 44.5 & $10 \mathrm{M}: 8 \mathrm{~F}$ & 8 (IQR: 4-12) & [43] \\
\hline Dai et al & China & 180 & NA & NA & 4.95 (SD: 2.46) & [44] \\
\hline Duan et al & China & 42 & 51.48 & $15 \mathrm{M}: 27 \mathrm{~F}$ & 7 (IQR: 2-13.5) & {$[45]$} \\
\hline Fang et al & China & 305 & 57 & 146M:159F & $6(\mathrm{R}: 1-15)$ & [46] \\
\hline Fu et al & China & 24 & 38.92 & 14M:10F & 5 (R: 0-35) & [47] \\
\hline Han et al & China & 226 & 44.4 & 97M:129F & 7 (IQR: 5-11) & [48] \\
\hline Jia et al & China & 44 & 46 & 15M: 29F & 6.28 (R: 1-14) & [49] \\
\hline Jiang et al & China & 43 & 50 & $15 \mathrm{M}: 28 \mathrm{~F}$ & 5.95 (R: 2-13) & [50] \\
\hline Jiang et al & China & 17 & - & $6 \mathrm{M}: 11 \mathrm{~F}$ & 7 (R: 1-14) & [51] \\
\hline \multirow[t]{2}{*}{ Jin et al. 1} & China & $21^{\mathrm{a}}$ & NA & NA & 4 (IQR: 3-7) & [52] \\
\hline & & $195^{b}$ & NA & NA & 5 (IQR: 3-8) & \\
\hline Lai et al & China & 330 & 47 & 161M:169F & 7 (IQR:4-12) & [53] \\
\hline Lai et al & China & 40 & NA & NA & 4.2 (IQR: 4.0-4.5) & [54] \\
\hline Li et al & China & 47 & 35 & $23 \mathrm{M}: 24 \mathrm{~F}$ & 9 (R: 1-20) & [55] \\
\hline Li et al & China & 30 & 32.57 & $30 F$ & 7.07 (SD: 4.08) & [56] \\
\hline Li et al & China & 86 & - & - & 6.3 (R: 1-12) & [57] \\
\hline Li et al & China & 51 & - & - & 5 (R: 2-10) & [58] \\
\hline Liu et al & China & 201 & 45.2 & 103M:98F & 6.3 (R: 1-20) & [59] \\
\hline Liu et al & China & 87 & NA & NA & $10.4(R: 2-25)$ & [60] \\
\hline Liu et al & China & 41 & 42 & $19 \mathrm{M}: 22 \mathrm{~F}$ & 8.8 (SD: 4.8) & [61] \\
\hline Liu et al & China & 44 & - & - & 8 (R: 1-19) & [62] \\
\hline Liu et al & China & 70 & 40 & 1.13 & $6(\mathrm{R}: 1-14)$ & [63] \\
\hline Lu et al & China & 100 & 37.13 & $52 \mathrm{M}: 48 \mathrm{~F}$ & 11.14 (SD: 6.05) & [64] \\
\hline Luo et al & China & 24 & 35.3 & 14M:10F & 8.5 (R: 1-14) & [65] \\
\hline $\mathrm{Ng}$ et al & China & 158 & 45 & $84 \mathrm{M}: 74 \mathrm{~F}$ & $5.5(S D: 3.26)$ & [66] \\
\hline Ping et al & China & 90 & - & - & 6.05 (IQR: 3.81-9.59) & [67] \\
\hline She et al & China & 991 & 43.96 & 1.16 & $5.2(S D: 1.9)$ & [68] \\
\hline Shen et al & China & 10 & 60.5 & $6 \mathrm{M}: 4 \mathrm{~F}$ & 4.5 (IQR: 2-6.5) & [69] \\
\hline Tian et al & China & 262 & 47.5 & 127M: 135F & 6.7 (SD: 5.2) & [70] \\
\hline Wang et al & China & 483 & NA & NA & 7 (IQR: 4-11) & [71] \\
\hline Wang et al & China & 275 & 49 & 128M: 147F & 6 (IQR: 3-9) & [72] \\
\hline Wang et al & China & 14 & NA & NA & 3 (IQR:2-5.25) & [73] \\
\hline Wang et al & China & 12 & - & - & 7.5 (IQR: 6-13.5) & [74] \\
\hline Wu et al & China & 41 & 38 & $19 \mathrm{M}: 22 \mathrm{~F}$ & 6.05 (SD: 3.84) & {$[75]$} \\
\hline Xia et al & China & 10 & 56.5 & $6 \mathrm{M}: 4 \mathrm{~F}$ & 7 (SD: 2.59) & [76] \\
\hline Yu et al & China & 132 & NA & 172M: 161F & 7.2 (IQR: 6.4-7.9) & [77] \\
\hline Yuan et al & China & 41 & 41 & 25M:16F & 6.5 (IQR: 4.0-11.5) & [78] \\
\hline Zhao et al & China & 136 & 49 & NA & 6 (IQR: 4-11) & [79] \\
\hline Zhang et al & China & 353 & - & - & 5.9 (IQR: 3.8-8.6) & [80] \\
\hline Zhong et al & China & 48 & 41 & $31 \mathrm{M}: 17 \mathrm{~F}$ & 6.86 (SD: 3.57) & [81] \\
\hline Zhu et al & China & 245 & - & - & 6 (R: 1-23) & [82] \\
\hline Chun et al & Korea & 35 & NA & NA & 2.87 (SD: 1.64) & [3] \\
\hline Viego et al & Argentina & 15 & NA & NA & 7.86 (SD: 6.38) & [24] \\
\hline Wong et al & Brunei & 135 & 36 & $82 / 53 \mathrm{~F}$ & 5 (R: 1-11) & [25] \\
\hline Pongpirul et al & Thailand & 83 & NA & NA & 5 (IQR: 3-8) & [26] \\
\hline Calba et al & France & 10 & NA & NA & 4.5 (IQR: 2-7) & [27] \\
\hline
\end{tabular}


Table 1 (continued)

\begin{tabular}{|c|c|c|c|c|c|c|}
\hline Author & Country & Case number & Age, years & Gender & Incubation period, days & References \\
\hline Laval et al & France & 23 & - & - & $4(\mathrm{R}: 1-13)$ & {$[28]$} \\
\hline Bui et al & Vietnam & 19 & NA & NA & $6.4(S D: 5.8)$ & [29] \\
\hline Böhm et al & Germany & 256 & NA & NA & $4.6(S D: 3.0)$ & [30] \\
\hline Patrikar et al & India & 268 & 36.45 & 162M:106F & 6.93 (SD: 5.87) & {$[32]$} \\
\hline Tan et al & Singapore & 164 & 44.2 & $77 \mathrm{M}: 87 \mathrm{~F}$ & 5 (SD: 2.3) & [34] \\
\hline
\end{tabular}

$\mathrm{R}$, range; IQR, interquartile range; $S D$, standard deviation; $M$, male; F, female; NA, Not available

${ }^{\text {a }}$ With gastrointestinal symptom

${ }^{\mathrm{b}}$ Without gastrointestinal symptom

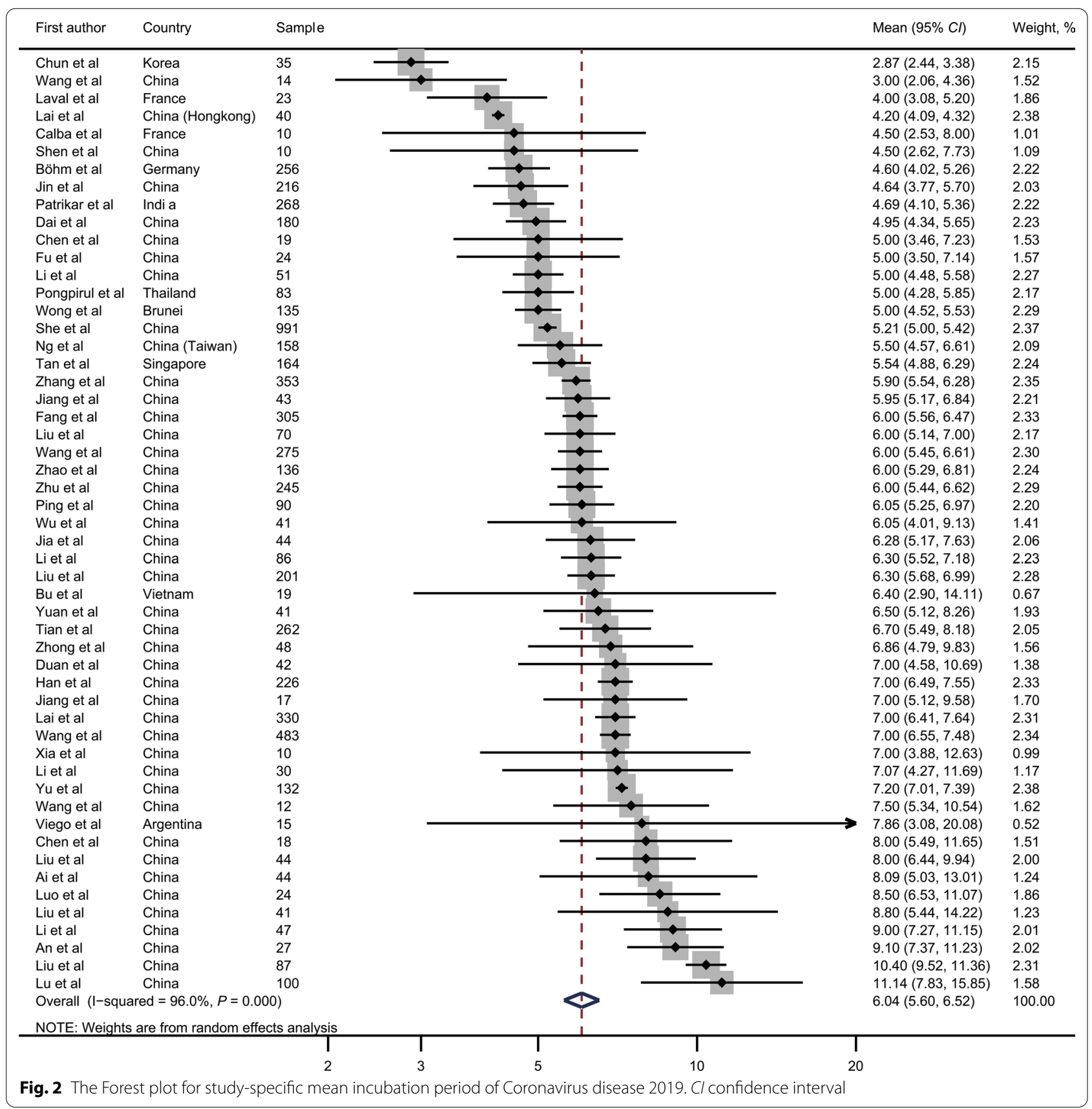


Table 2 The study-specific incubation period of Coronavirus disease 2019 in the meta-analysis

\begin{tabular}{|c|c|c|c|c|c|c|}
\hline Subgroup & $\begin{array}{l}\text { Number of } \\
\text { articles }\end{array}$ & $\begin{array}{l}\text { Number of } \\
\text { patients }\end{array}$ & $\begin{array}{l}\text { Mean incubation } \\
\text { period }(95 \% \mathrm{Cl})\end{array}$ & $I^{2}(\%)$ & $P_{\text {her }}$ & $P_{\text {reg }}$ \\
\hline \multicolumn{6}{|l|}{ Region } & $<0.001$ \\
\hline The mainland of China & 41 & 5459 & $6.5(6.1-6.9)$ & 90.7 & $<0.001$ & \\
\hline Outside the mainland of China & 12 & 1206 & $4.6(4.1-5.1)$ & 83.3 & $<0.001$ & \\
\hline \multicolumn{6}{|l|}{ Median or mean age (years) } & 0.807 \\
\hline$<40$ & 8 & 669 & $6.3(5.9-6.8)$ & 80.3 & $<0.001$ & \\
\hline$\geq 40$ & 21 & 3442 & $6.6(5.3-8.3)$ & 87.5 & $<0.001$ & \\
\hline \multicolumn{6}{|l|}{ Ratio of male to female } & 0.572 \\
\hline$\leq 1$ & 15 & 1999 & $6.5(6.0-7.1)$ & 78.6 & $<0.001$ & \\
\hline$>1$ & 15 & 2125 & $6.3(5.6-7.2)$ & 93.7 & $<0.001$ & \\
\hline \multicolumn{6}{|l|}{ Sample of study } & 0.691 \\
\hline$<100$ & 33 & 1249 & $6.1(5.3-7.1)$ & 95.0 & $<0.001$ & \\
\hline$\geq 100$ & 20 & 5416 & $5.9(5.5-6.4)$ & 93.9 & $<0.001$ & \\
\hline \multicolumn{6}{|c|}{ Estimation method of the date of infection } & 0.045 \\
\hline The earliest of exposure & 19 & 3130 & $5.7(5.0-6.4)$ & 96.0 & $<0.001$ & \\
\hline Exposure interval & 4 & 603 & $4.7(3.1-7.0)$ & 98.3 & $<0.001$ & \\
\hline Others & 30 & 2932 & $6.6(6.0-7.2)$ & 90.3 & $<0.001$ & \\
\hline \multicolumn{6}{|c|}{ Estimation method of the date of onset symptoms } & 0.064 \\
\hline The date of onset symptoms & 23 & 3411 & $5.6(5.0-6.3)$ & 97.7 & $<0.001$ & \\
\hline Other & 30 & 3254 & $6.4(5.8-7.1)$ & 91.0 & $<0.001$ & \\
\hline \multicolumn{6}{|l|}{ Estimation method of incubation period } & 0.090 \\
\hline From the earliest exposure to onset & 16 & 2296 & $5.7(5.0-6.5)$ & 95.7 & $<0.001$ & \\
\hline From the exposure interval to onset & 4 & 603 & $4.7(3.1-7.0)$ & 98.3 & $<0.001$ & \\
\hline Others & 33 & 3766 & $6.4(5.9-7.0)$ & 90.5 & $<0.001$ & \\
\hline \multicolumn{6}{|l|}{ Language } & 0.025 \\
\hline English & 31 & 4354 & $5.7(5.1-6.3)$ & 97.5 & $<0.001$ & \\
\hline Chinese & 22 & 2311 & $6.6(6.1-7.3)$ & 81.4 & $<0.001$ & \\
\hline
\end{tabular}

$P_{\text {heter }}$ for heterogeneity within each subgroup estimated by the Cochrane $\mathrm{Q}$ test

$P_{\text {reg }}$ for heterogeneity between subgroups using meta-regression analyses

$\mathrm{Cl}$, Confidence interval

of COVID-19 was 6.0 days globally and near 7.0 days in China. The findings of the meta-analysis and observation study were consistent in the Chinese population. Lu et al. reported a mean incubation period of 7.2 days (95\% CI 6.9-7.5) in 1158 patients with COVID-19, who also used the interval-censored data [84]. Their result was consistent with the result of this study. The reported mean incubation period varied significantly in previous studies, affecting the estimation method and study population. Some studies had a longer mean incubation period compared with this study, which used the earliest exposure date to estimate the incubation period. For example, Xiao et al. showed a mean incubation period of about 8 days, similar to the findings of Qin et al. [6,9]. Conversely, other studies that used the last exposure date reported a shorter mean incubation period than this study, ranging from 3 to 5 days $[8,85,86]$. The incubation period was overestimated using the date of the earliest exposure and underestimated using the date of the last exposure date primarily because of the inaccurate date of infection. In addition, studies with shorter maximum incubation periods had shorter mean incubation periods. Tan et al. reported a mean incubation period of 5 days (range: 1-12 days) in 164 patients with COVID-19 [34]. Similarly, a mean incubation period of 5 days (range: 1-11 days) was reported in 135 Brunei patients [25]. On the contrary, a longer mean incubation period was reported in studies with a broader range of incubation period $[5,60]$. The distribution type may have a negligible effect on the estimated incubation period of COVID-19, which was relatively robust among different distributions, including Log-normal distribution, Gamma distribution, Weibull distribution, and Erlang distribution [12]. Large samples and accurate data were important for understanding the incubation period. Therefore, our study provided a reliable incubation 
Table 3 The incubation period of Coronavirus disease 2019 in the 11545 patients and in the 218 patients

\begin{tabular}{|c|c|c|c|c|c|c|}
\hline & \multicolumn{2}{|l|}{11545 patients } & \multirow[t]{2}{*}{$P$} & \multicolumn{2}{|l|}{218 patients } & \multirow[t]{2}{*}{$P$} \\
\hline & N (\%) & $\begin{array}{l}\text { Mean incubation } \\
\text { period }(95 \% C l)\end{array}$ & & $\mathrm{N}(\%)$ & $\begin{array}{l}\text { Mean incubation } \\
\text { period }(95 \% \mathrm{Cl})\end{array}$ & \\
\hline Total & 11545 (100\%) & $7.1(7.0-7.2)$ & & $218(100 \%)$ & $6.8(6.2-7.4)$ & \\
\hline Gender & & & 0.603 & & & 0.145 \\
\hline Male & $5814(52.7 \%)$ & $7.2(7.0-7.4)$ & & $121(56.3 \%)$ & $6.3(5.4-7.2)$ & \\
\hline Female & $5215(47.3 \%)$ & $7.1(6.9-7.3)$ & & $94(43.7 \%)$ & $7.5(6.7-8.4)$ & \\
\hline Age, years & & & $<0.001$ & & & 0.009 \\
\hline$<18$ & $460(4.4 \%)$ & $8.6(8.0-9.3)$ & & $6(2.8 \%)$ & $9.3(5.3-16.3)$ & \\
\hline $18-40$ & $3789(36.3 \%)$ & $7.2(7.0-7.4)$ & & $73(34.1 \%)$ & $7.7(6.6-9.0)$ & \\
\hline $41-60$ & $4425(42.3 \%)$ & $7.1(6.9-7.2)$ & & $98(45.8 \%)$ & $5.7(4.9-6.6)$ & \\
\hline$>60$ & $1777(17.0 \%)$ & $7.0(6.6-7.3)$ & & $37(17.3 \%)$ & $8.0(6.5-9.8)$ & \\
\hline The date of onset & & & 0.319 & & & \\
\hline Reported date of onset ${ }^{a}$ & $7303(63.2 \%)$ & $7.1(7.0-7.3)$ & & & & \\
\hline Date of first treatment minus 2.5 days ${ }^{b}$ & $2168(18.8 \%)$ & $7.0(6.7-7.3)$ & & & & \\
\hline Date of diagnosis minus 5.4 days $^{c}$ & $2074(18.0 \%)$ & $7.3(7.0-7.6)$ & & & & \\
\hline
\end{tabular}

$\mathrm{N}$, number; $\mathrm{Cl}$, Confidence interval

a The incubation period was estimated in 7303 patients with the clear date of onset

${ }^{b}$ The incubation period was estimated in 2168 patients using the date of first treatment to fill in the missing date of onset

'The incubation period was estimated in 2074 patients using the date of diagnosis to fill in the missing date of onset

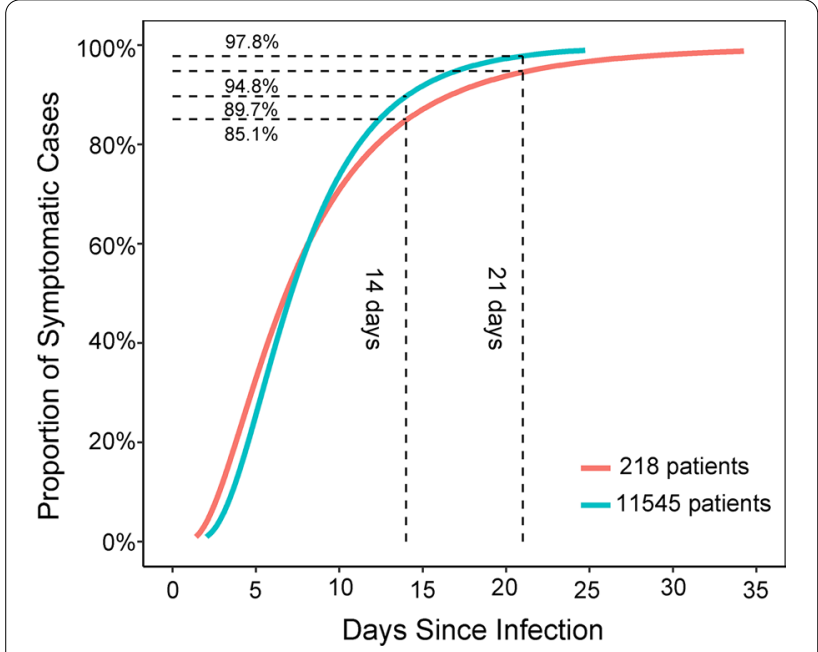

Fig. 3 The fitted distribution of incubation period of Coronavirus disease 2019

period of COVID-19, which was robust evidence for understanding SARS-CoV-2 transmission.

Given its potential impact on quarantine strategy, particular attention should be paid to the right tail end of the incubation period [2]. The 14-day quarantine strategy faced a challenge because the incubation period of COVID-19 exceeded 14 days [87]. In this study, among 11545 patients, more than $10 \%$ developed the disease 14 days after infection in the 11545 patients. Consistent

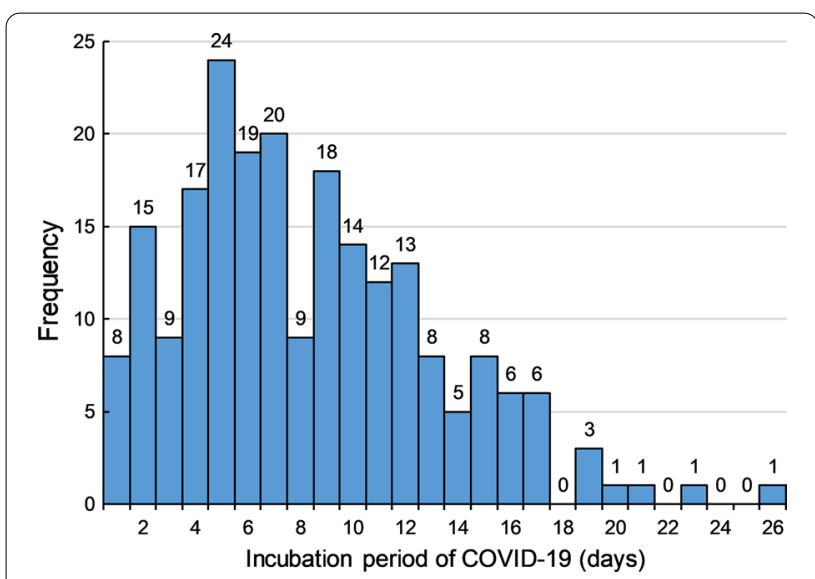

Fig. 4 The histogram of individual incubation period of Coronavirus disease 2019 in the 218 patients with precise data

with the results in 218 patients with precise data, the most extended incubation period was 26 days, and 27 patients had an incubation period of more than 14 days. Based on available evidence, the most extended incubation period of COVID-19 was 34 days in Shanghai's patients, far more than 14 days [4]. The extreme tail end of the incubation period may be affected by the sample size, the knowledge of SARS-CoV-2, and the observation period. Previous studies indicated that $5-10 \%$ of patients infected with SARS-CoV-2 had an incubation period longer than 14 days [88]. The above-mentioned evidence indicated that an extended quarantine period was needed 
to prevent the spread of SARS-CoV-2. When the quarantine interval was longer, more patients will be identified, and the epidemic will be controlled faster. Our findings suggested that the 21-day quarantine strategy will reduce the number of patients without symptoms by $80 \%$ compared with the 14-day quarantine strategy.

Take Wuhan as an example, after implementing a strict quarantine strategy of all residents on January 23, the number of new cases with COVID-19 decreased rapidly, with a $50 \%$ reduction after 14 days (February 6 ) and a $75 \%$ reduction after 28 days (February 20) [89]. However, the impact of the nucleic acid test on the development of quarantine strategies remained unknown. The nucleic acid test played a vital role in the prevention and control of the COVID-19 pandemic. A previous study reported that $8 \%$ of patients had a negative report of nucleic acid test after being quarantined for 14 days [90]. Cai et al. found that over $5 \%$ of patients had an incubation period of more than 14 days, which defined the incubation period as the interval from the earliest exposure to laboratory confirmation of COVID-19 or onset of symptoms and signs [91]. We hypothesized that, if only relying on clinical symptoms or nucleic acid test results, then the 14-day quarantine strategy will result in a part of patients not being recognized as confirmed cases. Therefore, adopting a 21-day quarantine strategy was recommended, particularly for places with insufficient detection resources or a high risk of being infected with SARS-CoV-2, such as contacting the patients infected with SARS-CoV-2 or coming from cities or countries where COVID-19 was epidemic.

In addition, the potential patients should be quarantined as soon as possible. We found that about $15 \%$ of patients had an incubation period of fewer than 3 days. In India, $25 \%$ of patients had an incubation period of fewer than 3 days (25th percentile: 3.0 days) [32]. Onethird of patients in Singapore had an incubation period of less than or equal to 3 days [35]. Patients with COVID19 were infectious before they developed symptoms [92]. Our study found that half of the patients developed symptoms within 7 days after infection. A meta-analysis showed that the mean serial interval of COVID-19 was 5.5 days [93]. Therefore, for COVID-19, the serial interval was shorter than the incubation period. We hypothesized that the spread of SARS-CoV-2 occurred on average 1.5 days before the onset of the disease. If all close contacts are quarantined on the 3rd day after infection, then more than $15 \%$ of the people infected with SARS-CoV-2 in close connections may have infected others. When COVID-19 patients and their close and sub-close contacts were quarantined for the first time, further transmission will be terminated.
The incubation period of COVID-19 varied with age. We found that the incubation period was different among age groups. In 218 patients, the incubation period presented a U-shaped curve with increasing age. The middle-aged group (41-60 years) had the shortest incubation period among the other groups, particularly the elderly group ( $\geq 61$ years) and those aged $18-40$ years. A similar age-specific distribution of incubation period was reported in the previous study with 136 patients, showing the shortest incubation period in patients aged 45 to 59 years [7]. Another study with 2555 patients also found a U-shaped curve distribution of incubation period in patients [6]. The mechanism of the effect of age on the COVID-19 incubation period was unclear. Possible explanations include a less intense immune response, a delay in the onset of symptoms, and a shorter exposure time and exposure rate in the elderly and children.

Focusing on the incubation period among different SARS-CoV-2 variants is important. Our finding showed that the incubation period in the mainland of China was longer than that outside the mainland of China (6.5 days vs 4.6 days, $P<0.001)$. Phylogenetic network analysis revealed that the types of SARS-CoV-2 were different among Chinese, Europeans, and Americans in the early stage of the COVID-19 pandemic [94]. Therefore, we hypothesized that the incubation period of COVID-19 may be different among SARS-CoV-2 variants. Recently, an outbreak of SARS-CoV-2 variant B.1.617.2 occurred in Guangzhou, China. In this outbreak, the mean incubation period was 4.4 days (95\% CI 3.9-5.0), which was shorter than that previously reported in China [95]. These evidences indicated that mutations affected the incubation period of COVID-19. However, more research was needed to explore the relationship of incubation period of COVID-19 with SARS-CoV-2 variants.

However, no difference in incubation period was observed between males and females. The previous study suggested that male was more susceptible to COVID19 , which may be due to the high plasma concentration of ACE2 [96]. However, our study did not observe a difference in incubation period by gender. Nie et al. also showed an insignificant difference in incubation period by gender ( 5 days vs 4 days; $P=0.22$ ) [8]. Yang et al. presented a similar conclusion [97]. The evidence indicated that gender maybe not a factor affecting the incubation period of COVID-19.

Several limitations should be stated in the present study. First, the current meta-analysis did not include studies published in languages other than Chinese or English because of language restrictions. Most of the included studies were from China. Secondly, information bias may also exist. As far as we know, this study had the largest sample size to date, but a part of patients was 
missing the date of onset. Although no significant difference in incubation periods was observed among the three estimation methods of the date of onset, the estimated date of onset may be biased from the actual date of onset. In addition, most patients had a doubly interval-censored data rather than precise date of infection. For extreme exposure intervals, we limited the exposure interval to 14 days, which might neglect a small part of the extreme incubation period. Third, in the 218 patients with precise data, the proportion of incubation period exceeding 14 days was higher than that in previous studies. This result would obtain an extreme right tail for the incubation period of COVID-19. Finally, the active contact tracing and testing (nucleic acid testing and antibody testing) may truncate the time between exposure to identifying infected persons. Therefore, caution should be taken when formulating quarantine strategies because the estimation of the incubation period did not involve nucleic acid tests and antibody tests.

\section{Conclusions}

This study provides evidence on the incubation period of COVID-19 to understand the transmission of disease and formulate preventive measures. The mean incubation period is 6.0 days globally, but it is longer in the mainland of China ( 6.5 days) than in other regions ( 4.6 days). The region- or age-specific incubation period should be paid attention to. Moreover, $10 \%$ of patients had an incubation period over 14 days in Chinese population, suggesting that the 14-day quarantine period may not be enough.

\begin{abstract}
Abbreviations
ACE2: Angiotensin-converting enzyme 2; Cl: Confidence interval; COVID-19: Coronavirus disease 2019; CNKI: China National Knowledge Infrastructure; IQR: Interquartile range; 2019-nCoV: Novel coronavirus 2019; NCP: Novel coronavirus pneumonia; MOOSE: Meta-analysis of observational studies in epidemiology; R: Range; RT-PCR: Reverse Transcription-Polymerase Chain Reaction; SD: Standard deviation; SARS-CoV-2: Severe acute respiratory syndrome coronavirus 2.
\end{abstract}

\section{Supplementary Information}

The online version contains supplementary material available at https://doi. org/10.1186/s40249-021-00901-9.

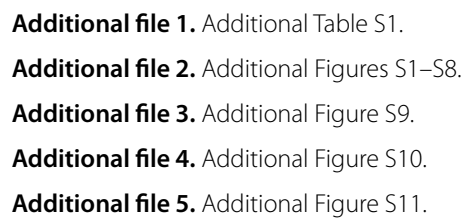

Acknowledgements

The authors would like to thank everyone who supported this study, especially thank Professor Junyuan Yang, Professor Xichao Duan, and Professor Xuezhi Li.

\section{Authors' contributions}

SC and CC designed and implemented this research; CC wrote the manuscript; JG, PZ, MY, and RL collected and screened individual information on COVID-19 patients; CC, DZ, and DD analyzed the data; JX made some suggestions and polished the language; SC provided advice for the revision of the paper; GD initiated this project, interpreted the results, and revised the manuscript. All authors read and approved the final manuscript.

\section{Funding}

The work was funded by the National Natural Science Foundation of China (NO.82073618 and NO.82002147); the Key Scientific Research Projects in Colleges and Universities of Henan Province [20A330004]; the National Science and Technology Specific Projects (NO.2018ZX10301407).

\section{Availability of data and materials}

The data used for this article is available from the corresponding author on reasonable request.

\section{Declarations}

Ethics approval and consent to participate

This study was approved by the Zhengzhou University Medical Ethics Committee (Zhengzhou, China). The information on all cases was collected from publicly available sources, and consent to participate was waived.

\section{Consent for publication}

Not applicable.

\section{Competing interests}

The authors declare that they have no competing interests.

\section{Author details}

${ }^{1}$ Department of Epidemiology and Health Statistics, College of Public Health, Zhengzhou University, No. 100 Kexue Avenue, Zhengzhou 450001, Henan, People's Republic of China. ${ }^{2}$ Department of Nutrition and Food Hygiene, College of Public Health, Zhengzhou University, No. 100 Kexue Avenue, Zhengzhou 450001, Henan, People's Republic of China. Infection Prevention and Control Department, The Fifth Affiliated Hospital of Zhengzhou University, No.3 Kangfuqian Street, Zhengzhou 450052, Henan, People's Republic of China. ${ }^{4}$ Henan Key Laboratory of Molecular Medicine, Zhengzhou University, No. 100 Kexue Avenue, Zhengzhou 450001, Henan, People's Republic of China. ${ }^{5}$ Centre for Biostatistics and Clinical Trials (BaCT), Peter MacCallum Cancer Centre, No. 305 Grattan Street, Melbourne 3000, Victoria, Australia.

Received: 17 March 2021 Accepted: 2 September 2021

Published online: 17 September 2021

\section{References}

1. World Health Organization. COVID-19 weekly epidemiological update (2 March 2021). https://www.who.int/publications/m/item/weekly-epide miological-update-2-march-2021. Accessed 4 Mar 2021.

2. Li ZY, Zhang Y, Peng LQ, Gao RR, Jing JR, Wang JL, et al. Demand for longer quarantine period among common and uncommon COVID-19 infections: a scoping review. Infect Dis Poverty. 2021;10:56.

3. Chun JY, Baek G, Kim Y. Transmission onset distribution of COVID-19. Int J Infect Dis. 2020;99:403-7.

4. Xie S, Zhang G, Yu H, Wang J, Wang S, Tang G, et al. The epidemiologic and clinical features of suspected and confirmed cases of imported 2019 novel coronavirus pneumonia in north Shanghai, China. Ann Transl Med. 2020;8:637.

5. You C, Deng Y, Hu W, Sun J, Lin Q, Zhou F, et al. Estimation of the timevarying reproduction number of COVID-19 outbreak in China. Int J Hyg Environ Health. 2020;228:113555.

6. Xiao Z, Xie X, Guo W, Luo Z, Liao J, Wen F, et al. Examining the incubation period distributions of COVID-19 on Chinese patients with different travel histories. J Infect Dev Ctries. 2020;14(4):323-7.

7. Kong TK. Longer incubation period of coronavirus disease 2019 (COVID19) in older adults. Aging Med (Milton). 2020;3(2):102-9. 
8. Nie X, Fan L, Mu G, Tan Q, Wang M, Xie Y, et al. Epidemiological characteristics and incubation period of 7015 confirmed cases with coronavirus disease 2019 outside Hubei Province in China. J Infect Dis. 2020;222:26-33.

9. Qin J, You C, Lin Q, Hu T, Yu S, Zhou XH. Estimation of incubation period distribution of COVID-19 using disease onset forward time: a novel crosssectional and forward follow-up study. Sci Adv. 2020;6:eabc1202.

10. Ren X, Li Y, Yang X, Li Z, Cui J, Zhu A, et al. Evidence for pre-symptomatic transmission of coronavirus disease 2019 (COVID-19) in China. Influ Other Respir Viruses. 2021;15:19-26.

11. Lauer SA, Grantz KH, Bi Q, Jones FK, Zheng Q, Meredith HR, et al. The incubation period of coronavirus disease 2019 (COVID-19) from publicly reported confirmed cases: estimation and application. Ann Intern Med. 2020;172:577-85.

12. Huang $S, L i J$, Dai C, Tie Z, Xu J, Xiong X, et al. Incubation period of coronavirus disease 2019: new implications for intervention and control. Int Environ Health Res. 2021. https://doi.org/10.1080/09603123.2021.19057 81.

13. Li Q, Guan $X$, Wu P, Wang $X$, Zhou L, Tong Y, et al. Early transmission dynamics in Wuhan, China, of novel coronavirus-infected pneumonia. N Engl J Med. 2020;382:1199-207.

14. Guo CX, He L, Yin JY, Meng XG, Tan W, Yang GP, et al. Epidemiological and clinical features of pediatric COVID-19. BMC Med. 2020;18:250.

15. Stroup DF, Berlin JA, Morton SC, Olkin I, Williamson GD, Rennie D, et al, Meta-analysis of observational studies in epidemiology: a proposal for reporting. Meta-analysis Of Observational Studies in Epidemiology (MOOSE) group. JAMA. 2000;283:2008-12.

16. Xu L, Chen N, Zhang X, Chen Z. An evaluation of statistical, NMME and hybrid models for drought prediction in China. J Hydrol (Amst). 2018;566:235-49.

17. Hozo SP, Djulbegovic B, Hozo I. Estimating the mean and variance from the median, range, and the size of a sample. BMC Med Res Methodol. 2005;5:13.

18. Zhang J, Litvinova M, Wang W, Wang Y, Deng X, Chen X, et al. Evolving epidemiology and transmission dynamics of coronavirus disease 2019 outside Hubei province, China: a descriptive and modelling study. Lancet Infect Dis. 2020;20:793-802.

19. National Health Commission of China. Chinese National COVID-19 Diagnosis and Treatment Plan (Trial Eight Edition). 2021. http://www.nhc.gov. cn/yzygj/s7653p/202104/7de0b3837c8b4606a0594aeb0105232b.shtml. Accessed 25 Aug 2021. (in Chinese)

20. Reich NG, Lessler J, Cummings DA, Brookmeyer R. Estimating incubation period distributions with coarse data. Stat Med. 2009;28:2769-84.

21. Linton NM, Kobayashi T, Yang Y, Hayashi K, Akhmetzhanov AR, Jung SM, et al. Incubation period and other epidemiological characteristics of 2019 novel coronavirus infections with right truncation: a statistical analysis of publicly available case data. J Clin Med. 2020;9:538.

22. Sartwell PE. The distribution of incubation periods of infectious disease. 1949. Am J Epidemiol. 1995;141:386-94.

23. Huang QQ, Ritchie SC, Brozynska M, Inouye M. Power, false discovery rate and Winner's Curse in eOTL studies. Nucleic Acids Res. 2018:46:e133.

24. Viego V, Geri M, Castiglia J, Jouglard E. Incubation period and serial interval of Covid-19 in a chain of infections in Bahia Blanca (Argentina). Cien Saude Colet. 2020;25:3503-10.

25. Wong J, Chaw L, Koh WC, Alikhan MF, Jamaludin SA, Poh WWP, et al. Epidemiological Investigation of the First 135 COVID-19 cases in Brunei: implications for surveillance, control, and travel restrictions. Am J Trop Med Hyg. 2020;103:1608-13.

26. Pongpirul WA, Wiboonchutikul S, Charoenpong L, Panitantum N, Vachiraphan A, Uttayamakul S, et al. Clinical course and potential predictive factors for pneumonia of adult patients with Coronavirus Disease 2019 (COVID-19): a retrospective observational analysis of 193 confirmed cases in Thailand. PLoS Negl Trop Dis. 2020;14:e0008806.

27. The SARS-CoV-2 Variant with Lineage B.1.351 Clusters Investigation Team. Linked transmission chains of imported SARS-CoV-2 variant B.1.351 across mainland France, January 2021. Euro Surveill. 2021;26:2100333.

28. de Laval F, Grosset-Janin A, Delon F, Allonneau A, Tong C, Letois F, et al. Lessons learned from the investigation of a COVID-19 cluster in Creil, France: effectiveness of targeting symptomatic cases and conducting contact tracing around them. BMC Infect Dis. 2021;21:457.
29. Bui LV, Nguyen HT, Levine H, Nguyen HN, Nguyen TA, Nguyen TP, et al. Estimation of the incubation period of COVID-19 in Vietnam. PLOS ONE. 2020;15:e0243889.

30. Bohm S, Woudenberg T, Chen D, Marosevic DV, Bohmer MM, Hansen L, et al. Epidemiology and transmission characteristics of early COVID-19 cases, 20 January-19 March 2020, in Bavaria, Germany. Epidemiol Infect. 2021;149:e65.

31. Bohmer MM, Buchholz U, Corman VM, Hoch M, Katz K, Marosevic DV, et al. Investigation of a COVID-19 outbreak in Germany resulting from a single travel-associated primary case: a case series. Lancet Infect Dis. 2020;20:920-8.

32. Patrikar SR, Kotwal A, Bhatti VK, Banerjee A, Chatterjee K, Kunte R, et al. Incubation period and reproduction number for novel coronavirus 2019 (COVID-19) infections in India. Asia Pac J Public Health. 2020;32:458-60.

33. Vivian Thangaraj JW, Murhekar M, Mehta Y, Kataria S, Brijwal M, Gupta $\mathrm{N}$, et al. A cluster of SARS-CoV-2 infection among Italian tourists visiting India, March 2020. Indian J Med Res. 2020;151:438-43.

34. Tan WYT Wong LY, Leo YS, Toh M. Does incubation period of COVID-19 vary with age? A study of epidemiologically linked cases in Singapore. Epidemiol Infect. 2020;148:e197.

35. Pung R, Chiew CJ, Young BE, Chin S, Chen MI, Clapham HE, et al. Investigation of three clusters of COVID-19 in Singapore: implications for surveillance and response measures. Lancet. 2020;395:1039-46.

36. Lee H, Kim K, Choi K, Hong S, Son H, Ryu S. Incubation period of the coronavirus disease 2019 (COVID-19) in Busan, South Korea. J Infect Chemother. 2020;26:1011-3.

37. Ki M, Task Force for -nCo V. Epidemiologic characteristics of early cases with 2019 novel coronavirus (2019-nCoV) disease in Korea. Epidemiol Health. 2020;42:e2020007.

38. Alwafi H, Naser AY, Qanash S, Brinji AS, Ghazawi MA, Alotaibi B, et al. Predictors of length of hospital stay, mortality, and outcomes among hospitalised COVID-19 patients in Saudi Arabia: a cross-sectional study. J Multidiscip Healthc. 2021;14:839-52.

39. Ario AR, Mirembe BB, Biribawa C, Bulage L, Kadobera D, Wamala R. Timing of onset of symptom for COVID-19 from publicly reported confirmed cases in Uganda. Pan Afr Med J. 2021;38:168.

40. Ai J, Wang Y, Chen J, Liu X, Fan W, Huang G, et al. The cross-sectional study of hospitalized COVID-19 patients in Xiangyang. Hubei province. New Med. 2020;30:355-63 (in Chinese).

41. An R, Chen Z, Ayidar B, Nurahamat D, Wang Y, Liu ZQ, et al. Analysis of clinical characteristics of COVID - 19 infection pneumonia in lli area. Xinjiang Med J. 2021;51(170-73):69.

42. Chen M, Jia HJ, Wang H, Zheng X, Wang Q. Infection status of coronavirus disease 2019 cases and close contacts in Fuzhou. Fujian. Dis Surveill. 2020;35:608-12 (in Chinese).

43. Chen G, Wu MZ, Qin CJ, Wu BB, Luo WR, Liu L, et al. Epidemiological analysis of 18 patients with COVID-19. Eur Rev Med Pharmacol Sci. 2020;24:12522-6.

44. Dai J, Yang $L$, Zhao J. Probable longer incubation period for elderly COVID-19 cases: analysis of 180 contact tracing data in Hubei Province, China. Risk Manag Healthc Policy. 2020;13:1111-7.

45. Duan Y, Jiang R, Kang W, Zhao A, Huang M. Clinical features of patients with new coronavirus pneumonia admitted to ICU in Shanxi province. J Shanxi Med Univ. 2020;51:852-7 (in Chinese).

46. Fang D, Ma JY, Guan J, Wang M, Song Y, Tian D, et al. Manifestations of digestive system of hospitalized patients with coronavirus disease 2019 in Wuhan, China: a single-center descriptive study. Chin J Dig. 2020;40:151-6 (in Chinese).

47. Fu Y, Meng Z, Yu G, Fu K, Liu A, Hu JH, et al. Analysis of epidemiology and clinical characteristics in 24 patients with COVID-19 in Liuzhou. Intern Med China. 2020;15:392-5 (in Chinese).

48. Han T, Hua L, He S, Zhou Y, Hou C, Chen J, et al. The epidemiological characteristics of cluster transmission of coronavirus disease 2019 (COVID-19): a multi-center study in Jiangsu Province. Am J Transl Res. 2020;1 12:6434-44

49. Jia J, Hu X, Yang F, Song X, Dong L, Zhang J, et al. Epidemiological characteristics on the clustering nature of COVID-19 in Qingdao City, 2020: a descriptive analysis. Disaster Med Public Health Prep. 2020;14:643-7.

50. Jiang G, Wang C, Song L, Wang X, Zhou Y, Fei C, et al. Aerosol transmission, an indispensable route of COVID-19 spread: case study of a department-store cluster. Front Environ Sci Eng. 2021;15:46. 
51. Jiang Y, Huang J, Zhong Y, Li Y, Wei S, Huang S, et al. Epidemiological investigation on a cluster epidemic of COVID-19. Mod Prev Med. 2020;47:3807-10 (in Chinese).

52. Jin X, Lian JS, Hu JH, Gao J, Zheng L, Zhang YM, et al. Epidemiological, clinical and virological characteristics of 74 cases of coronavirusinfected disease 2019 (COVID-19) with gastrointestinal symptoms. Gut. 2020;69:1002-9.

53. Lai C, Yu R, Wang M, Xian W, Zhao X, Tang Q, et al. Shorter incubation period is associated with severe disease progression in patients with COVID-19. Virulence. 2020;11:1443-52.

54. Lai CKC, Ng RWY, Wong MCS, Chong KC, Yeoh YK, Chen Z, et al. Epidemiological characteristics of the first 100 cases of coronavirus disease 2019 (COVID-19) in Hong Kong Special Administrative Region, China, a city with a stringent containment policy. Int J Epidemiol. 2020;49:1096-105.

55. Li X, Geng X, Zhao X, Liu L, Wang W, Cui L, et al. A comprehensive analysis of 47 COVID-19 cases in Jinan City. J Shandong Univ (Health Sci). 2020;58:23-7 (in Chinese).

56. Li C, Guo H, Zheng Q, Huang B, He F, Xu J, et al. Analysis of clinical features and outcomes of young female patients with mild COVID-19. Int J Respir. 2020;40:978-81 (in Chinese).

57. Li X, Zhang W, Li J, An S, Xiang R, Zhang M, et al. Epidemiological characteristics of coronavirus disease 2019 in the elderly in Shandong Province. Chin J Mult Organ Dis Elder. 2020;19:191-4. (in Chinese)

58. Li X, M. L, Zhou L, Li M, Z M, Xiang R, et al. Epidemiological analysis of corona virus disease 2019 in southern Shandong region. Chin J Integr Tradit West Med Intensive Crit Care. 2020;27:35-8. (in Chinese)

59. Liu P, Niu R, Chen J, Tang Y, Tang W, Xu L, et al. Epidemiological and clinical features in patients with coronavirus disease 2019 outside of Wuhan, China: special focus in asymptomatic patients. PLoS Negl Trop Dis. 2021;15:e0009248.

60. Liu H, Gao J, Wang Y, Jie J, Luo J, Xu Y, et al. Epidemiological and clinical characteristics of 2019 novel coronavirus disease (COVID-19) in Jilin, China: a descriptive study. Medicine (Baltimore). 2020;99:e23407.

61. Liu L, Du Y, Bai JY, Li J, Luo Y, Wang L, et al. Analysis of early characteristics of patients with novel coronavirus infection in Kunming City, Yunnan Province. China. Chin J Zoonoses. 2020;36(424-26):28 (in Chinese).

62. Liu L, Jing L, Li Y, Tian Y, You J, Cui J, et al. Clustering of 2019 novel coronavirus disease cases in Liaoning province:reported data-base analysis. Chin J Public Health. 2020;36:473-6. (in Chinese)

63. Liu G, Wang X, Lu JA, Zhao X, Lei Z, Li J, et al. The epidemic characteristics of uovel coronavirus infected pneumonia in Uingxia Hui Autonomous Region. J Ningxia Med Univ. 2020;42:109-13. (in Chinese)

64. Lu W, Zhou L, Chen W, Xu R, Li Y, Zhang P. Clinical characteristics of coronavirus disease 2019 in Dongguan: a retrospective study. Hainan Med J. 2021;32:85-7. (in Chinese)

65. Luo L, Go D. Epidemiological analysis of COVID-19 outbreak in Bazhong City in 2020. Parasitoses Infect Dis. 2020;18:117-20. (in Chinese)

66. Ng TC, Cheng HY, Chang HH, Liu CC, Yang CC, Jian SW, et al. Comparison of estimated effectiveness of case-based and population-based interventions on COVID-19 containment in Taiwan. JAMA Intern Med. 2021;181:913-21.

67. Ping K, Lei M, Gou Y, Tao Z, Yao G, Hu C, et al. Epidemiologic characteristics of COVID-19 in Guizhou Province, China. J Infect Dev Ctries. 2021;15:389-97.

68. She K, Zhang D, Qi C, Liu T, Jia Y, Zhu Y, et al. Epidemiological characteristics and incubation period of coronavirus disease 2019 in Anhui Province. J Shandong Univ (Health Sci). 2020;58:44-52. (in Chinese)

69. Shen J, Yu J, Yan Y, Sang Y, Qin L, Na M, et al. Clinical and chest HRCT characteristics in family group outbreak of novel coronavirus pneumonia. J Dalian Med Univ. 2020;42:32-6. (in Chinese)

70. Tian S, Hu N, Lou J, Chen K, Kang X, Xiang Z, et al. Characteristics of COVID-19 infection in Beijing. J Infect. 2020;80:401-6.

71. Wang P, Lu JA, Jin Y, Zhu M, Wang L, Chen S. Statistical and network analysis of 1212 COVID-19 patients in Henan, China. Int J Infect Dis: IJID. 2020;95:391-8.

72. Wang Y, Liao B, Guo Y, Li F, Lei C, Zhang F, et al. Clinical characteristics of patients infected with the novel 2019 coronavirus (SARS-Cov-2) in Guangzhou, China. Open Forum Infect Dis. 2020;7:ofaa187.

73. Wang X, Zhou Q, He Y, Liu L, Ma X, Wei X, et al. Nosocomial outbreak of COVID-19 pneumonia in Wuhan, China. Eur Respir J. 2020;55:2000544.
74. Wang W, Zhou S, Ying J, Xie D, Zhang Q, Sun Y. Epidemiological characteristics of cluster outbreaks of COVID-19 in Neijiang. South China J Prev Med. 2020:46:243-6. (in Chinese)

75. Wu Y, Zhuang X, Huang H, Lin YT. Epidemiological and clinical characteristics of 41 cases of coronavirus disease 2019. Chin J Integr Tradit West Med Intensive Crit Care. 2020;27:390-3. (in Chinese)

76. Xia XY, Wu J, Liu HL, Xia H, Jia B, Huang WX. Epidemiological and initial clinical characteristics of patients with family aggregation of COVID-19. J Clin Virol. 2020;127:104360.

77. Yu X, Sun X, Cui P, Pan H, Lin S, Han R, et al. Epidemiological and clinical characteristics of 333 confirmed cases with coronavirus disease 2019 in Shanghai. China Transbound Emerg Dis. 2020;67:1697-707.

78. Yuan X, Qin D, Tian F, Che F, Zheng Q, Heng X. Epidemiological and clinical characteristics of patient with COVID-19. J Mol Imaging. 2020;43:3816. (in Chinese)

79. Zhao C, Xu Y, Zhang X, Zhong Y, Long L, Zhan W, et al. Public health initiatives from hospitalized patients with COVID-19. China J Infect Public Health. 2020;13:1229-36.

80. Zhang T, Ding S, Zeng Z, Cheng H, Zhang C, Mao X, et al. Estimation of incubation period and serial interval for SARS-CoV-2 in Jiangxi, China, and an updated meta-analysis. J Infect Dev Ctries. 2021;15:326-32.

81. Zhong ZF, Huang J, Yang X, Peng JL, Zhang XY, Hu Y, et al. Epidemiological and clinical characteristics of COVID-19 patients in Hengyang, Hunan Province, China. World J Clin Cases. 2020;8:2554-65.

82. Zhou Y, Ming J, Liu S. Epidemiologic characteristics and double-buffered strategy for patients in orthopedic surgery during the novel coronavirus outbreak: Wuhan's experience. Eur J Med Res. 2021;26:39.

83. Zhu N, Chen S, Yang GP, Ning S, Li X, Nian Y, et al. Analysis of the path from discovery of novel coronavirus disease 2019 cases to its diagnosis in Shaanxi Province. J Xi'an Jiaotong Univ (Med Sci). 2020;41:923-6. (in (hinese)

84. Lu QB, Zhang Y, Liu MJ, Zhang HY, Jalali N, Zhang AR, et al. Epidemiological parameters of COVID-19 and its implication for infectivity among patients in China, 1 January to 11 February 2020. Euro Surveill. 2020;25:2000250.

85. Yang $N$, Shen Y, Shi C, Ma AHY, Zhang $X$, Jian X, et al. In-flight transmission cluster of COVID-19: a retrospective case series. Infect Dis (Lond). 2020;52:891-901.

86. Shi P, Gao Y, Shen Y, Chen E, Chen H, Liu J, et al. Characteristics and evaluation of the effectiveness of monitoring and control measures for the first 69 Patients with COVID-19 from 18 January 2020 to 2 March in Wuxi, China. Sustain Cities Soc. 2021;64:102559.

87. Cimolai N. In pursuit of the right tail for the COVID-19 incubation period. Public Health. 2021;194:149-55.

88. Bikbov B, Bikbov A. Maximum incubation period for COVID-19 infection: do we need to rethink the 14-day quarantine policy? Travel Med Infect Dis. 2021;40:101976.

89. Pan A, Liu L, Wang C, Guo H, Hao X, Wang Q, et al. Association of public health interventions with the epidemiology of the COVID-19 outbreak in Wuhan, China. JAMA. 2020;323:1915-23.

90. Dickens BL, Koo JR, Lim JT, Sun H, Clapham HE, Wilder-Smith A, et al. Strategies at points of entry to reduce importation risk of COVID-19 cases and reopen travel. J Travel Med. 2020;27:taaa141.

91. Cai Y, Liu J, Yang H, Wang M, Guo Q, Huang D, et al. Association between incubation period and clinical characteristics of patients with COVID-19. J Int Med Res. 2020;48:300060520956834.

92. Guan Q, Liu M, Zhuang YJ, Yuan Y, Wang SS, Li J, et al. Epidemiological investigation of a family clustering of COVID-19. Zhonghua Liu Xing Bing Xue Za Zhi. 2020;41:629-33. (in Chinese)

93. Hussein M, Toraih E, Elshazli R, Fawzy M, Houghton A, Tatum D, et al. Meta-analysis on serial intervals and reproductive rates for SARS-CoV-2. Ann Surg. 2020;273:416-23.

94. Forster P, Forster L, Renfrew C, Forster M. Phylogenetic network analysis of SARS-CoV-2 genomes. Proc Natl Acad Sci USA. 2020;117:9241-3.

95. Zhang M, Xiao J, Deng A, Zhang Y, Zhuang Y, Hu T, et al. Transmission dynamics of an outbreak of the COVID-19 Delta variant B.1.617.2Guangdong Province, China, May-June 2021. China CDC Weekly. 2021;7:584-6.

96. Sama IE, Ravera A, Santema BT, van Goor H, Ter Maaten JM, Cleland JGF, et al. Circulating plasma concentrations of angiotensin-converting 
enzyme 2 in men and women with heart failure and effects of reninangiotensin-aldosterone inhibitors. Eur Heart J. 2020;41:1810-7.

97. Yang L, Dai J, Zhao J, Wang Y, Deng P, Wang J. Estimation of incubation period and serial interval of COVID-19: analysis of 178 cases and
131 transmission chains in Hubei province, China. Epidemiol Infect. 2020;148:e117.
Ready to submit your research? Choose BMC and benefit from:

- fast, convenient online submission

- thorough peer review by experienced researchers in your field

- rapid publication on acceptance

- support for research data, including large and complex data types

- gold Open Access which fosters wider collaboration and increased citations

- maximum visibility for your research: over $100 \mathrm{M}$ website views per year

At BMC, research is always in progress.

Learn more biomedcentral.com/submissions 